\title{
IgA-kappa Type Multiple Myeloma Affecting Proximal and Distal Renal Tubules
}

\author{
Kesami Minemura, Kazuo Ichikawa, Nobuo Itoh*, Naomi Suzuki, Masahiro Hara, Satoshi Shigematsu, \\ Hiroaki KobaYashi, Kunihide Hiramatsu and Kiyoshi Hashizume
}

\begin{abstract}
A 45-year-old male was admitted because of chest pain, lumbago, and bilateral ankle pain. Examination disclosed hypophosphatemic osteomalacia, acquired Fanconi syndrome, and abnormalities in distal nephron such as distal renal tubular acidosis and renal diabetes insipidus. Further exploration revealed IgA kappa multiple myeloma excreting urinary Bence Jones protein (kappa-light chain). Renal biopsy revealed thick basement membranes and electron-dense crystals in proximal tubular epithelial cells. Immunofluorescent studies revealed deposition of kappalight chain in renal tubular epithelial cells that caused the renal tubular damage. Although the osteomalacia was relieved by medical treatment, the urinary Bence Jones protein and the renal tubular defects were not improved by the chemotherapy for the myeloma. The patient died of exacerbation of multiple myeloma at 50 years of age.

(Internal Medicine 40: 931-935, 2001)
\end{abstract}

Key words: hypophosphatemic osteomalacia, Bence Jones protein, renal diabetes insipidus, fanconi syndrome, distal renal tubular acidosis

\section{Introduction}

Multiple myeloma frequently impairs renal function $(1,2)$. The most frequent renal disorder is myeloma kidney in which renal failure usually develops. Although the adult Fanconi syndrome is a well-known complication, it is an uncommon renal manifestation of multiple myeloma (3); fifty-seven such cases have been reported to date (4). Clinical manifestations of adult Fanconi syndrome involve type II renal tubular acidosis, renal glycosuria, aminoaciduria, phosphaturia, and renal hypouricemia $(2,3)$. It is frequently associated with electron-dense deposits of reabsorbed immunoglobulin light chains in the proximal renal tubular epithelium $(1,5-7)$. They are usually kappa-light chains, but rare instances of Fanconi syndrome caused by lambda-light chains have been described (8). In the present report, we describe a case of impaired proximal and distal renal tubular functions (acquired Fanconi syndrome, renal diabetes insipidus, and distal renal tubular acidosis) and hypophosphatemic osteomalacia.

\section{Case Report}

A 45-year-old man was admitted to our department because of chest pain, lumbago, and bilateral ankle pain on September 23,1990 . At 14 years of age, he had rheumatic fever. Thereafter he did not have any trouble until 40 years of age when he noticed exertional dyspnea and palpitation. He visited another hospital and was told to have mitral stenosis, aortic regargitation, and atrial fibrillation. Nocturia, polyuria, and polydipsia were also noticed. At 43 years of age, he had proteinuria and renal glycosuria. At 44 years of age, he had pain in the chest, lower back, and bilateral ankles. As the chest pain and lumbago increased, he visited the orthopedics department of our hospital where the elevation of serum Al-p and low serum level of phosphorus were noticed. He was referred to our department because of possible hypophosphatemic osteomalacia. On physical examination, his height was $165 \mathrm{~cm}$, body weight $62 \mathrm{~kg}$. Blood pressure was $120 / 80 \mathrm{mmHg}$, pulse rate $96 / \mathrm{min}$ with irregular rhythm, and body temperature $36.0^{\circ} \mathrm{C}$. Spontaneous pain that deteriorated upon physical movement was noticed in the anterior chest, lower back, and bilateral ankles. He had tenderness and knock pain in the bilateral clavicles and upper ribs. Bone X-ray examination revealed marked osteoporosis in the vertebrae and Looser's zone in the pelvic bone. Bone scintigraphy using ${ }^{99 \mathrm{~m}} \mathrm{Tc}$-hydroxymethylenediphosphonate revealed multiple accumulations in the bilateral costal bones, lumbar vertebrae, and bilateral ankles. Laboratory examination (Table 1) revealed elevation of serum Al-p 3 and creatinine, and low levels of serum phosphorus and uric acid. Urinalysis showed proteinuria and glucosuria despite normal blood glucose. Daily urinary output was increased due to renal diabetes insipidus. Arterial blood gas analysis showed metabolic acidosis with

From the Department of Aging Medicine and Geriatrics and *the Second Department of Pathology, Shinshu University School of Medicine, Matsumoto Received for publication May 24, 2000; Accepted for publication April 23, 2001

Reprint requests should be addressed to Dr. Kazuo Ichikawa, the Department of Aging Medicine and Geriatrics, Shinshu University School of Medicine, 3-11 Asahi, Matsumoto, Nagano 390-8621 
Minemura et al

Table 1. Laboratory Findings

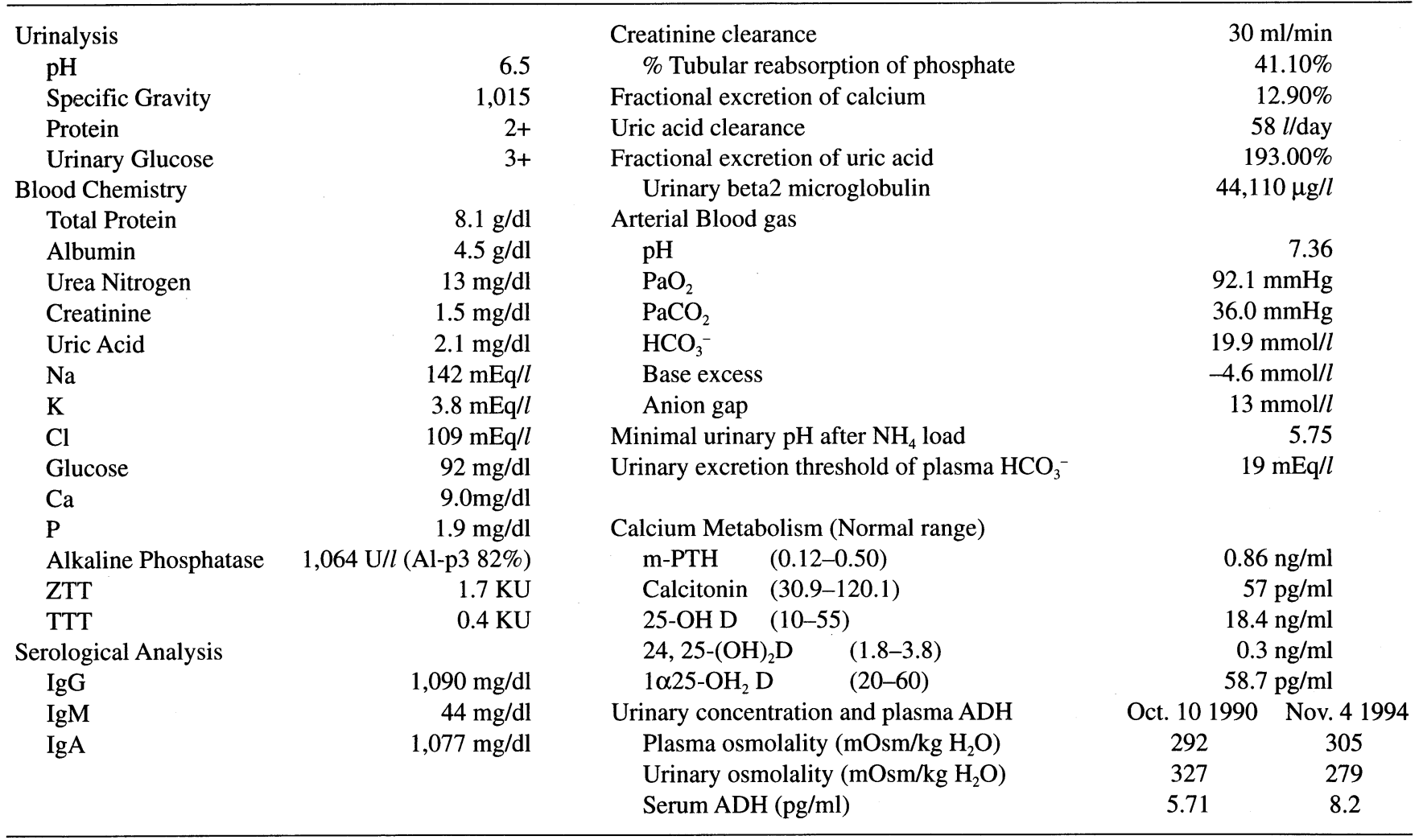

normal anion gap. Renal failure and impairment of proximal renal tubular reabsorption of phosphorus, calcium, and uric acid were present. The elevation of urinary $\beta_{2}$ microglobulin also suggested impairment of proximal renal tubules. $\mathrm{NaHCO}_{3}$ loading test revealed the proximal renal tubular acidosis. These findings as well as generalized aminoaciduria led to the diagnosis of acquired Fanconi syndrome. Urinary acidification defect after $\mathrm{NH}_{4} \mathrm{Cl}$ loading indicated distal renal tubular acidosis as well. $1 \alpha$ hydroxylation of $25-\mathrm{OH}$ vitamin $\mathrm{D}$ was enhanced whereas 24 hydroxylation was diminished, reflecting the secondary hyperparathyroidism as evidenced by the high serum level of PTH. Electrophoresis of serum proteins showed no monoclonal peak, but the amount of beta globulin was increased. Immunoelectrophoresis of serum protein revealed a monoclonal increase of IgA with kappa light chain. Bence Jones protein (kappa light chain) was revealed on immunoelectrophoresis of urine. Aspiration biopsy of sternal bone marrow revealed $20 \%$ myeloma cells. Magnetic resonance imaging (MRI) showed multiple nodular lesions in the vertebral bodies. Renal biopsy specimen showed almost intact glomeruli with thick basement membrane of proximal renal tubules. Intracellular electron-dense crystals of proximal tubular cells were found (Fig. 1A, B). Immunofluorescent studies revealed that the crystals were composed of kappa light chain (Fig. 1C) and that no positive staining was obtained using antibodies against IgA, consistent with previous reports $(7,9)$. Administration of melphalan $(12 \mathrm{mg} /$ day $)$ and prednisolone (90 mg/day) (MP) was initiated from Sep- tember 17 for four days that was repeated monthly. Daily intramuscular administration of $300 \times 10^{4} \mathrm{IU}$ alpha interferon was also initiated. Administration of $2.5 \mu \mathrm{g}$ /day of 1 alpha $(\mathrm{OH})$ vitamin $\mathrm{D}$ and $20 \mathrm{U} /$ week of synthetic salmon calcitonin were also started. Chest pain, lumbago, and bilateral ankle pain decreased in two months. The MP therapy was continued at an out-patient clinic for about 4 years. Reduction of the serum IgA was incomplete and myeloma cells were 6-10\% of total cells in bone marrow aspiration biopsy specimen (Fig. 2). Administration of the alpha interferon was discontinued on November 3, 1991 due to thrombocytopenia that hampered the monthly administration of the MP. After discontinuation of the alpha interferon, the MP therapy was repeated monthly and serum IgA level was decreased to $95-110 \mathrm{mg} / \mathrm{dl}$ and bone marrow aspiration biopsy showed $2-4 \%$ myeloma cells. Urinary excretion of kappa-light chain, however, was not reduced throughout the entire course (Fig. 2). Sodium bicarbonate at 4 $\mathrm{g} /$ day and phosphorus equivalent of sodium/potassium phosphate buffer ( $1.8 \mathrm{~g} /$ day $)$ were also given for the treatment of the renal tubular acidosis and for the hypophosphatemic osteomalacia. Proximal and distal renal tubular functions were not altered after the treatment (data not shown). Bone mineral density of lumbar spine (L2-L4) was $0.685 \mathrm{~g} / \mathrm{cm}^{2}$ as determined antero-posteriorly by dual energy $\mathrm{X}$-ray absorptiometry on June $15,1994$.

He had been stable until October 12, 1994 when he was admitted to our hospital because of dyspnea, chest pain and 

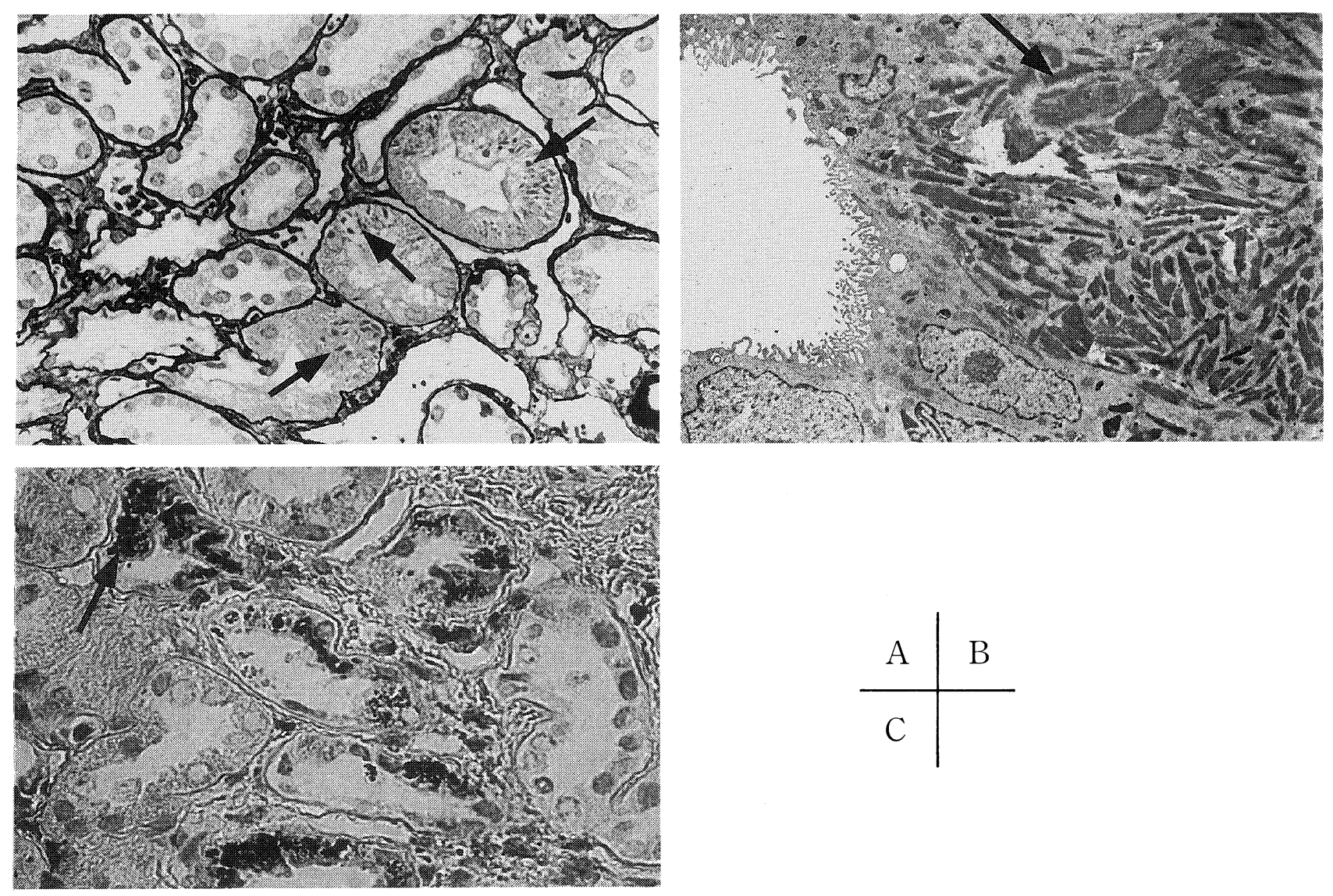

Figure 1. Microscopic examinations of renal biopsy specimens. Renal biopsy specimen revealed tubular or rod-like deposits (indicated by arrows) in proximal tubular epithelium (A) (PASM, $\times 160)$ that was electron-dense on Electronmicroscopy (B) $(\times 1,830)$. Renal tubular deposits were positively stained with antibodies against kappa-light chain using peroxidase as a marker (C) ( $\times 160)$. PASM: periodic acid silver methenamine stain.

left hip pain. On admission, he had congestive heart failure and lung edema without evidence of acute myocardial infarction. Palpation of the head showed multiple firm tumors in the skull which were manifested as punched-out lesions in skull $\mathrm{X}$-ray. MRI revealed increased numbers and size of plasmacytomas in the vertebral bodies and a large mass in the proximal part of the left femur. In spite of local irradiation to the tumor, he finally developed subtrochanteric fracture of the left femur on October 29, 1994. Due to renal diabetes insipidus, hypoosmolar polyuria persisted despite increased plasma osmolarity and appropriate elevation of plasma ADH levels (Table 1). Regime of the chemotherapy was changed to vincristine $(0.4$ $\mathrm{mg} /$ day) and adriamycin (13 mg/day), together with 4-day cycles of dexamethasone ( $40 \mathrm{mg} /$ day) without success. He died of recurrent multiple myeloma on December 29, 1994. Autopsy was not performed.

\section{Discussion}

We described a patient with hypophosphatemic osteomalacia and renal dysfunction caused by IgA kappa multiple my- eloma excreting urinary kappa-light chain. Proximal renal tubular acidosis, renal glycosuria, generalized aminoaciduria, phosphaturia with hypophosphatemic osteomalacia, and increased fractional excretion of uric acid with hypouricemia are full-blown symptoms of Fanconi syndrome $(2,3)$, and increased fractional excretion of calcium also indicates damage of the proximal renal tubules. Since the first report of the acquired Fanconi syndrome and multiple myeloma in 1954 (10), there have been several reports of such cases in the English literature $(6,11-14)$. Urinary acidification defects of distal nephron were not examined in these reports except one report (14) in which the authors found the absence of distal renal tubular acidosis. In this regard, the present case was unusual as the distal renal tubular acidosis was associated with Fanconi syndrome and multiple myeloma. Another evidence demonstrating dysfunctions of the distal nephron was the presence of acquired renal diabetes insipidus manifested as the persistent polyuria since the age of about 40 years and as the elevated plasma osmolality with copious hypoosmolar urine despite appropriate elevation of plasma ADH concentration (15) (Table 1). Neither amyloid deposition nor nephrocalcinosis was found in the 


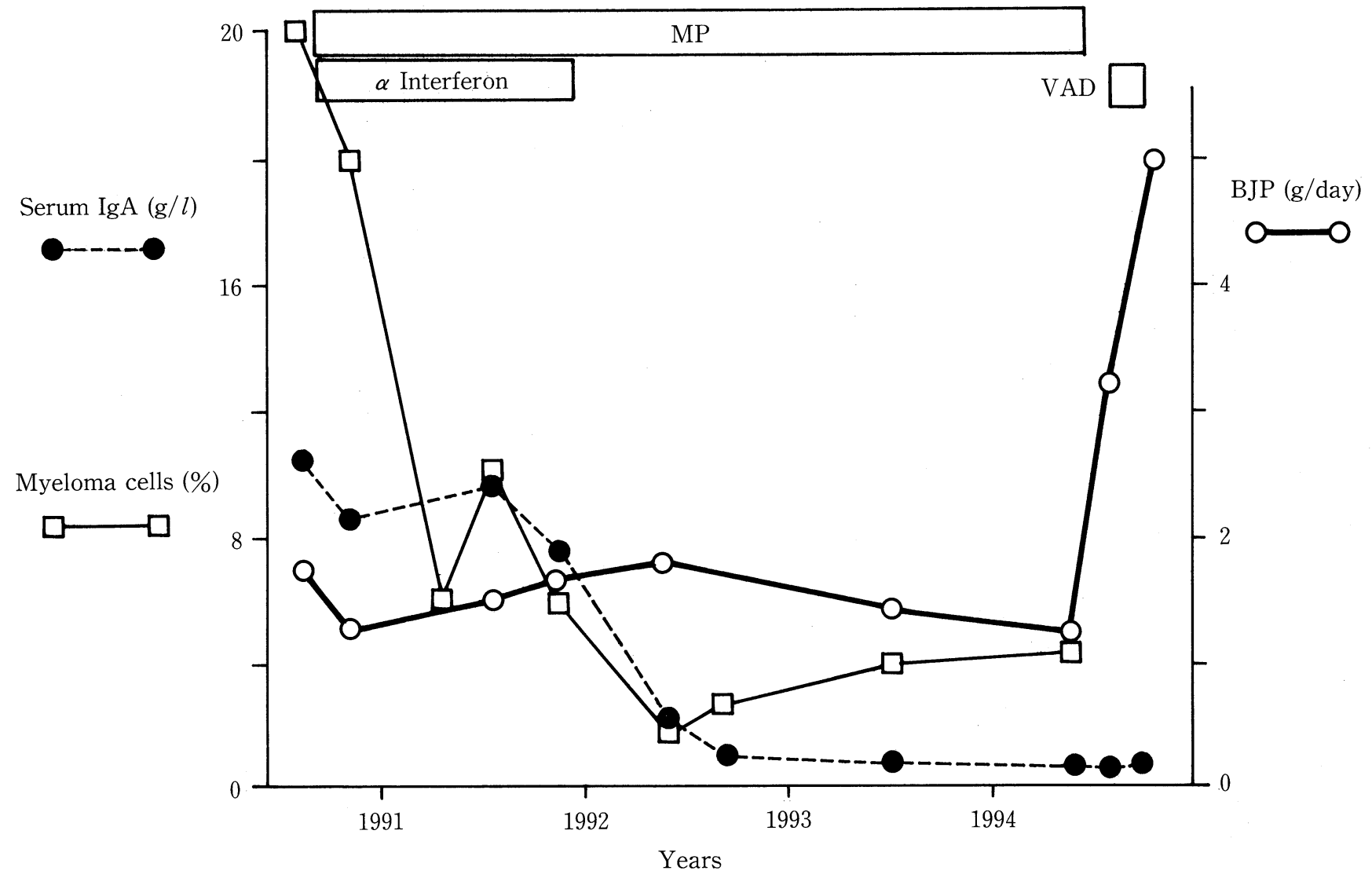

Figure 2. Clinical course of multiple myeloma. The changes in urinary excretion of Bence Jones protein, serum IgA, and myeloma cells in bone marrow aspiration biopsy specimen are shown during the treatment with melphalan and prednisolone, alpha interferon, and vincristine, adriamycin, and dexamethasone. BJP: Bence Jones protein, MP: Melphalan and prednisolone, VAD: vincristine; adriamycin and dexamethasone.

renal biopsy specimen.

Most cases of Fanconi syndrome, but not all (8), are associated with myeloma producing kappa type Bence Jones protein. It was considered that the electron dense deposits of reabsorbed immunoglobulin light chain in the proximal renal tubular epithelium cause aquired Fanconi syndrome (5-7). However in some cases of Fanconi syndrome, the proximal renal tubules appeared normal or showed only nonspecific changes and the intracellular protein crystals are not as specific for Fanconi syndrome as previously thought $(1,4)$. Current evidence suggests the variable domain of monoclonal light chains that are resistant to proteolysis due to peculiar amino acid sequences prevent catabolism by the tubular cells $(4,9)$ and impair tubular transport (16). So it would be the quality, not the quantity, of the Bence Jones protein that causes the renal tubular damage. Many reports (11-13) showed improvement of the Fanconi syndrome after successful treatment of the multiple myeloma leading to disappearance of Bence Jones protein. In one report (14), despite the treatment of the multiple myeloma for 3 years, hypouricemia and hypophosphatemia were not improved. In that report, however, whether the Bence Jones proteins were reduced by the treatment or not is unclear. In the present case, treatment of the multiple myeloma did not improve dysfunction of the renal tubules, probably because Bence Jones proteinuria persisted even after the MP therapy decreased the numbers of myeloma cells and the concentrations of serum IgA (Fig. 2). The present case supports the idea that Bence Jones protein causes proximal renal tubular damage and reduction of Bence Jones protein is essential for the improvement of Fanconi syndrome.

\section{References}

1) Morita T. Renal lesions in multiple myeloma and related disorders. Acta Medica et Biologica 43: 173-196, 1995.

2) Roth KS, Foreman JW, Segal S. The Fanconi syndrome and mechanisms of tubular transport dysfunction. Kidney Int 20: 705-716, 1981.

3) Colussi G, Barbarano L, Airaghi D, et al. Clinical spectrum of tubular disorders from light chains. in: The Kidney in Plasma Cell Dyscrasias. Minetti L, D'Amico C, Ponticelli C, Eds. Kluwer Academic, Dordrecht, 1988: 191-209.

4) Messiaen T, Deret S, Mougenot B, et al. Adult Fanconi syndrome secondary to light chain gammopathy. Clinicopathologic heterogeneity and unusual features in 11 patients. Medicine (Baltimore) 79: 135-154, 2000. 


\section{Renal Tubular Damage in Myeloma}

5) Maldonado JE, Velosa JA, Kyle RA, et al. Fanconi syndrome in adults. A manifestation of a latent form of myeloma. Am J Med 58: 354-364, 1975.

6) Chan KW, Ho FCS, Chan MK. Adult Fanconi syndrome in k light chain myeloma. Arch PatholLab Med 111: 139-142, 1987.

7) Truong LD, Mawad J, Cagle P, Mattioli C. Cytoplasmic crystals in multiple myeloma-associated Fanconi's syndrome. A morphological study including immunoelectron microscopy. Arch Pathol Lab Med 113: 781$785,1989$.

8) Isobe T, Kametani F, Shinoda T. V-domain deposition of lambda Bence Jones protein in the renal tubular epithelial cells in a patient with the adult Fanconi syndrome with myeloma. Amyloid 5: 117-120, 1998.

9) Aucouturier P, Bauens M, Khamlichi AA, et al. Monoclonal Ig L chain and $\mathrm{L}$ chain $\mathrm{V}$ domain fragment crystallization in myeloma-associated Fanconi's syndrome. J Immunol 150: 3561-3568, 1993.

10) Sirota TH, Hamerman D. Renal function studies in an adult subject with the Fanconi syndrome. Am J Med 16: 138-152, 1954.

11) Scheele C. Light chain myeloma with features of the adult Fanconi syndrome: Six year remission following one course of melphalan. Acta Med
Scand 199: 533-537, 1976.

12) Gailani S, Seon BK, Henderson ES. K light chain-myeloma associated with adult fanconi syndrome: response of the nephropathy to treatment of myeloma. Med Pediat Oncol 4: 141-147, 1978.

13) Uchida $S$, Matsuda $O$, Yokota $T$, et al. Adult Fanconi syndrome secondary to kappa-light chain myeloma: improvement of tubular function after treatment for myeloma. Nephron 55: 332-335, 1990.

14) Yonemura K, Matsushima H, Kato A, Isozaki T, Hishida A. Acqired Fanconi syndrome associated with IgG k multiple myeloma: observations on the mechanisms of impaired renal acid excretion. Nephrol Dial Transplant 12: 1251-1253, 1997.

15) Robertson GL, Berl T. Pathophysiology of water metabolism. in: The Kidney. $3^{\text {rd }}$ ed. Brenner BM, Rector Jr RC, Eds. WB Saunders Co. Philadelphia, 1986: 385-432.

16) Batuman V, Guan S, O'Donovan R, Puschett JB. Effect of myeloma light chains and glucose transport in renal proximal tubule cells. Ren Physiol Biochem 17: 294-300, 1994. 